\title{
Culture of mouse peritoneal macrophages with mouse serum induces lipid bodies that associate with the parasitophorous vacuole and decrease their microbicidal capacity against Toxoplasma gondii
}

\author{
Laura Azeredo Miranda Mota', João Roberto-Neto ${ }^{1,2}$, Verônica Gomes Monteiro', \\ Caroliny Samary Silva Lobato', Marco Antonio de Oliveiraa, ${ }^{1,3}$, Maura da Cunha', Heloisa D'Ávila ${ }^{2,4}$, \\ Sérgio Henrique Seabra ${ }^{5}$, Patrícia Torres Bozza ${ }^{2}$, Renato Augusto DaMatta ${ }^{1 /+}$
}

\begin{abstract}
${ }^{1}$ Laboratório de Biologia Celular e Tecidual, Universidade Estadual do Norte Fluminense, Campos dos Goytacazes, RJ, Brasil ${ }^{2}$ Laboratório de Imunofarmacologia, Fundação Oswaldo Cruz, Rio de Janeiro, RJ, Brasil '²aboratório de Parasitologia e Biotecnologia, Instituto de Pesquisa e Desenvolvimento, Universidade do Vale do Paraíba, São José dos Campos, SP, Brasil ${ }^{4}$ Laboratório de Biologia Celular, Universidade Federal de Juiz de Fora, Juiz de Fora, MG, Brasil ${ }^{5}$ Laboratório de Tecnologia em Bioquímica e Microscopia, Centro Universitário Estadual da Zona Oeste, Rio de Janeiro, RJ, Brasil
\end{abstract}

Lipid bodies [lipid droplets (LBS)] are lipid-rich organelles involved in lipid metabolism, signalling and inflammation. Recent findings suggest a role for LBs in host response to infection; however, the potential functions of this organelle in Toxoplasma gondii infection and how it alters macrophage microbicidal capacity during infection are not well understood. Here, we investigated the role of host LBs in T. gondii infection in mouse peritoneal macrophages in vitro. Macrophages cultured with mouse serum (MS) had higher numbers of LBs than those cultured in foetal bovine serum and can function as a model to study the role of LBs during intracellular pathogen infection. $L B s$ were found in association with the parasitophorous vacuole, suggesting that $\mathrm{T}$. gondii may benefit from this lipid source. Moreover, increased numbers of macrophage LBs correlated with high prostaglandin $E_{2}\left(P G E_{2}\right)$ production and decreased nitric oxide (NO) synthesis. Accordingly, LB-enriched macrophages cultured with MS were less efficient at controlling T. gondii growth. Treatment of macrophages cultured with MS with indomethacin, an inhibitor of PGE2 production, increased the microbicidal capacity against $\mathrm{T}$. gondii. Collectively, these results suggest that culture with MS caused a decrease in microbicidal activity of macrophages against $\mathrm{T}$. gondii by increasing PGE2 while lowering $N O$ production.

Key words: macrophages - lipid bodies - nitric oxide - prostaglandin - microbicidal capacity - Toxoplasma gondii

Macrophages, the most differentiated cells of the mononuclear phagocyte system, become activated after contact with pathogens or pathogen-derived molecules and molecules of the immune system. Classical activation enhances macrophage microbicidal capacity leading to the production of nitric oxide (NO) (Martinez et al. 2009). Macrophages with a high number of lipid bodies (LBs) (also known as lipid droplets) have been characterised as markers of cell activation and inflammation (Melo et al. 2006) and have been associated with infectious inflammatory diseases including parasitic infections (D’Ávila et al. 2011).

LBs are lipidic-rich organelles that are highly regulated and are present in all types of eukaryotic cells. LBs have well-characterised functions in lipid storage and metabolism. Additionally, LBs may be involved in other cellular processes such as vesicular trafficking (Beller et al. 2010) and inflammatory functions (Pacheco et al.

doi: 10.1590/0074-0276140119

Financial support: CNPq, FAPERJ, CAPES

+ Corresponding author: renato@uenf.br

Received 5 April 2014

Accepted 26 June 2014
2002, Bozza et al. 2009). Many studies relate the presence of this organelle with enhanced production of inflammatory mediators (Pacheco et al. 2002, D'Ávila et al. 2006) such as prostaglandin $\mathrm{E}_{2}\left(\mathrm{PGE}_{2}\right)$ (Bozza et al. 2011). In vivo and in vitro intracellular pathogen infections increase the number of LBs in macrophages through highly regulated mechanisms (D'Ávila et al. 2006, 2011, Samsa et al. 2009). The data gathered so far suggest that intracellular pathogens may take advantage of these LBs to support their own life cycle. However, little is known about the functions of this organelle and its influence on the microbicidal capacity of macrophages.

Lipids are important in the maintenance of many cellular structures and the survival of intracellular parasites. Protozoa are not able to synthesise cholesterol (Coppens 2006). Thus, the intracellular pathogen Toxoplasma gondii diverts a large variety of lipid precursors from the host cytoplasm and efficiently converts them into complex lipids (Nishikawa et al. 2005, Coppens 2006). Moreover, host cells have been demonstrated to be the major contributor of lipids to the intravacuolar network of the parasitophorous vacuole (PV) that harbours $T$. gondii (Caffaro \& Boothroyd 2011). The dependence on exogenous sources of cholesterol for the proper parasite growth implies that $T$. gondii has established mechanisms for acquiring, transporting and sorting this lipid (Coppens 2006), which has a direct impact on its development in- 
side the PV. This parasite has been observed to manage host organelles such as mitochondria and the endoplasmic reticulum around the PV to facilitate the incorporation of host elements that allow its survival and growth (Sinai \& Joiner 2001). Recently, T. gondii infection has been shown to induce LB biogenesis in skeletal muscle cells and this organelle is found in close contact to the PV membrane in the vacuolar matrix, which directly interacts with the parasite membrane, suggesting that LBs deliver their content to T. gondii (Gomes et al. 2014). In addition to the recruitment of the endoplasmic reticulum and mitochondria (Sinai \& Joiner 1997), T. gondii-infected macrophages may cause host cell LBs to associate with the PV, supporting the development of the parasite.

Macrophages are heterogeneous cells and their differences depend mainly on their microenvironment (Gordon \& Taylor 2005). Thus, the heterogeneity of macrophages in vitro can be studied by varying the culture conditions. The use of mouse serum (MS) as a culture supplement instead of foetal bovine serum (FBS) results in cells with different properties in systems such as bone marrow colony formation (Dahl \& Lindqvist 1989), dendritic cells (Salucci et al. 2005) and macrophages (Crocker et al. 1988). The aim of this paper is to report the effect of culturing macrophages with MS and how their microbicidal capacity against $T$. gondii changes.

\section{MATERIALS AND METHODS}

Animals and peritoneal macrophages - Swiss and $\mathrm{C} 3 \mathrm{H} / \mathrm{HeJ}$ mice were obtained from North Fluminense State University (UENF) and Oswaldo Cruz Foundation animal facility, respectively. Peritoneal washes were performed on male mice (25-30 g) using Hank's solution and the resident macrophages were seeded on glass coverslips in 24-well plates triphenyl phosphate (TPP). After $1 \mathrm{~h}$ at $37^{\circ} \mathrm{C}$ in a $5 \% \mathrm{CO}_{2}$ atmosphere, cells were washed and cultured in Dulbecco's modified Eagle's medium (DMEM) (Sigma) with 2\% FBS (Life Technologies) or 2\% MS (Crocker et al. 1988, Monteiro et al. 2005).

Ethics statement - This study was conducted in strict accordance with the Brazilian law 11794/08. The animal studies protocol was reviewed and approved by the Committee on the Ethics of Animal Experiments of the UENF (protocol 87). Mice were euthanised with $\mathrm{CO}_{2}$ in strict accordance with the recommendations in the Guide for the Care and Use of Laboratory Animals of the National Institutes of Health. The authors declare that have no conflict of interests.

$M S$ - MS was obtained as previously described (Monteiro et al. 2005). Briefly, mouse blood was harvested by cardiac puncture and allowed to clot in the syringe and the serum was collected, centrifuged, inactivated, aliquoted and frozen at $-20^{\circ} \mathrm{C}$.

Macrophage spreading - After culture, macrophages were fixed using $4 \%$ formaldehyde (Sigma) in PBS for 10 min, washed, Giemsa (Sigma) stained, dehydrated and mounted using Entellan (Merck). For some analyses, macrophages were not stained. Morphological observations were performed using a $100 \times$ oil immersion objective under a Zeiss Axioplan microscope equipped with differential interference contrast microscopy, and the images captured using the AxioCam Mrc5, and the Axiovision program were processed using Photoshop. The surface area of macrophages in $\mu \mathrm{m}^{2}$ was calculated after integrating the measurements of images of 50 cells in the Analysis ${ }^{\mathbb{B}}$ software.

Evaluation of the acidic compartment and LBS and F4/80 labelling - Live macrophages were incubated at $37^{\circ} \mathrm{C}$ in a $5 \% \mathrm{CO}_{2}$ atmosphere with acridine orange (Sigma) (acidic compartment marker) in DMEM, washed, further incubated in DMEM, mounted with DMEM and observed. Formaldehyde-fixed macrophages were washed, incubated with Nile red (Sigma), oil red (Sigma), BODIPY 493/503 (Molecular Probes) and $\mathrm{OsO}_{4}$ (Sigma) (lipid markers) (Melo et al. 2011), washed and mounted in ProLong Gold with 4'-6-diamidino-2phenylindole (DAPI) (Molecular Probes). The acidic compartments and LBs of live macrophages were also double-labelled using acridine orange and BODIPY, respectively. Macrophages were incubated with $10 \mu \mathrm{g} / \mathrm{mg}$ of acridine orange for $30 \mathrm{~min}$, washed, incubated with $1 \mathrm{M}$ of BODIPY for $5 \mathrm{~min}$, washed and mounted over DMEM. Fixed macrophages were also washed, incubated with PBS containing 3\% bovine serum albumin (BSA), incubated with anti-F4/80 (A3-1, AbD Serotec) diluted 1:100 in PBS containing 3\% BSA, washed, incubated with anti-rat-Alexa 488 (Molecular Probes) diluted 1:100, washed and mounted in ProLong Gold. Cells were observed under a Zeiss Axioplan fluorescence microscope equipped with a 100 HBO mercury lamp. Images were captured and processed as before. The number of LBs per 100 macrophages was counted in triplicates in independent experiments.

Electron microscopy - For scanning electron microscopy (SEM), cells cultured on coverslips were fixed in $4 \%$ fresh formaldehyde, $2.5 \%$ glutaraldehyde (Sigma) in $0.1 \mathrm{M}$ cacodylate buffer (Sigma), $\mathrm{pH} 7.2$ for $1 \mathrm{~h}$. The cells were washed, post-fixed with $1 \% \mathrm{OsO}_{4}$ in $0.1 \mathrm{M}$ cacodylate buffer, dehydrated in ethanol, critical point dried in $\mathrm{CO}_{2}$, covered with a $20 \mathrm{~nm}$ gold film and observed using a Zeiss 962 Digital Scanning Electron Microscope.

For transmission electron microscopy (TEM), the cells were cultured in $25 \mathrm{~cm}^{2}$ culture flasks (TPP), washed, infected and fixed as above. The cells were washed in $0.1 \mathrm{M}$ imidazole (Sigma) buffer, $\mathrm{pH} 7.5$, postfixed with $2 \% \mathrm{OsO}_{4}$ in imidazole buffer and washed (Angermüller \& Fahimi 1982). Cells were dehydrated in graded acetone and embedded in epoxy resin (Sigma). Thin sections were stained with uranyl acetate (Sigma) and lead citrate (Sigma) and observed in a Zeiss EM900 Transmission Electron Microscope operated at $80 \mathrm{kV}$.

$L B$ association to the $P V-\mathrm{LB}$ association was first monitored by fluorescence microscopy after Nile red staining to detect LBs and DAPI for parasite localisation. The cells were observed under a fluorescence microscope. For TEM, LBs were localised using the imidazolebuffered osmium tetroxide method as described above.

Determination of NO production and PGE2 assay Mouse peritoneal macrophages were cultured for 1, 24 and $48 \mathrm{~h}$ in DMEM with $2 \%$ FBS or MS, washed and 
activated with $100 \mu \mathrm{g} / \mathrm{mL}$ lipopolysaccharide (Sigma) and $50 \mathrm{U} / \mathrm{mL}$ interferon- $\gamma$ (Sigma) in DMEM supplemented with the same serum. After $24 \mathrm{~h}$ of culture, supernatants from all three time points were collected and nitrite was measured by the Griess reagent (Sigma) as described previously (Seabra et al. 2004). The same supernatants were also assayed in duplicate by ELISA (Cayman Chemicals, USA) to measure $\mathrm{PGE}_{2}$ production as described by D'Ávila et al. (2006).

Microbicidal action of macrophages against T. gondii - Macrophage microbicidal capacity against $T$. gondii was assayed as previously described (Guillermo \& DaMatta 2004). T. gondii RH strain tachyzoites were obtained from the peritoneal cavities of infected mice (Seabra et al. 2004). Macrophages were cultured for $24 \mathrm{~h}$ in 24-well plates without coverslips and infected with $5 \times 10^{5} \mathrm{~T}$. gondii per well for $1 \mathrm{~h}$. Infected macrophages were washed, activated and cultured for five days. The adherent cells on the well bottom were scraped into $500 \mu \mathrm{L}$ DMEM using a yellow tip, homogenised and a $10 \mu \mathrm{L}$ sample from each well, in triplicate, was diluted 1:10 in 4\% formaldehyde in PBS. Tachyzoites were counted using a Neubauer chamber to assess their growth. For the $\mathrm{PGE}_{2}$ inhibitor study, macrophages were pre-treated with the non-steroidal antiinflammatory drug indomethacin (Sigma) $(1 \mu \mathrm{g} / \mathrm{mL}) 24 \mathrm{~h}$ before the infection and daily for five days. After that, the number of tachyzoites per well was determined.

Data analyses - The experimental results were expressed as the mean \pm standard deviation and comparisons were performed using Student's $t$ test. A p-value < 0.05 was considered statistically significant. Numbers of replicates are indicated in the Figure captions.

\section{RESULTS}

Culturing in MS induces the formation of LBs in peritoneal macrophages - Macrophage cultures containing with FBS (MoFBS) or MS (MoMS) resulted in morphological differences and a greater number of vesicle-like organelles in MoMS (Fig. 1A, B). The fibroblast-like appearance of MoMS was also confirmed by SEM (Fig. $1 C, D)$. Some regions of the plasma membrane of MoMS had fewer protrusions and were more homogenous and smoother and revealed round contours that suggest the presence of vesicle-like organelles beneath the membrane (Fig. 1D). MoMS occupied significantly more area than MoFBS (Fig. 1E). MoMS and MoFBS presented similar levels of F4/80 antigen on their surface (not shown).

The first approach to determine the nature of the vesicle-like organelles present in MoMS was to determine if these structures were acidic compartments by acridine orange labelling. MoMS had similar acidic compartments to MoFBS and the vesicle-like organelles induced by MS culture were not acridine orange labelled (Fig. 2). Next, the lipid-selective dyes oil red, BODIPY, Nile red and $\mathrm{OsO}_{4}$ were used to verify if these vesicle-like organelles were LBs. Lipid positive vesicles were observed in both macrophage types, but MoMS had more LBs than MoFBS (Fig. 3A-H). MoMS were double labelled with acridine orange and BODIPY and no colocalisation was observed in the vesicle-like organelles (Fig. 3I), clearly indicating their lipid composition. The lipidic nature of the vesicles was
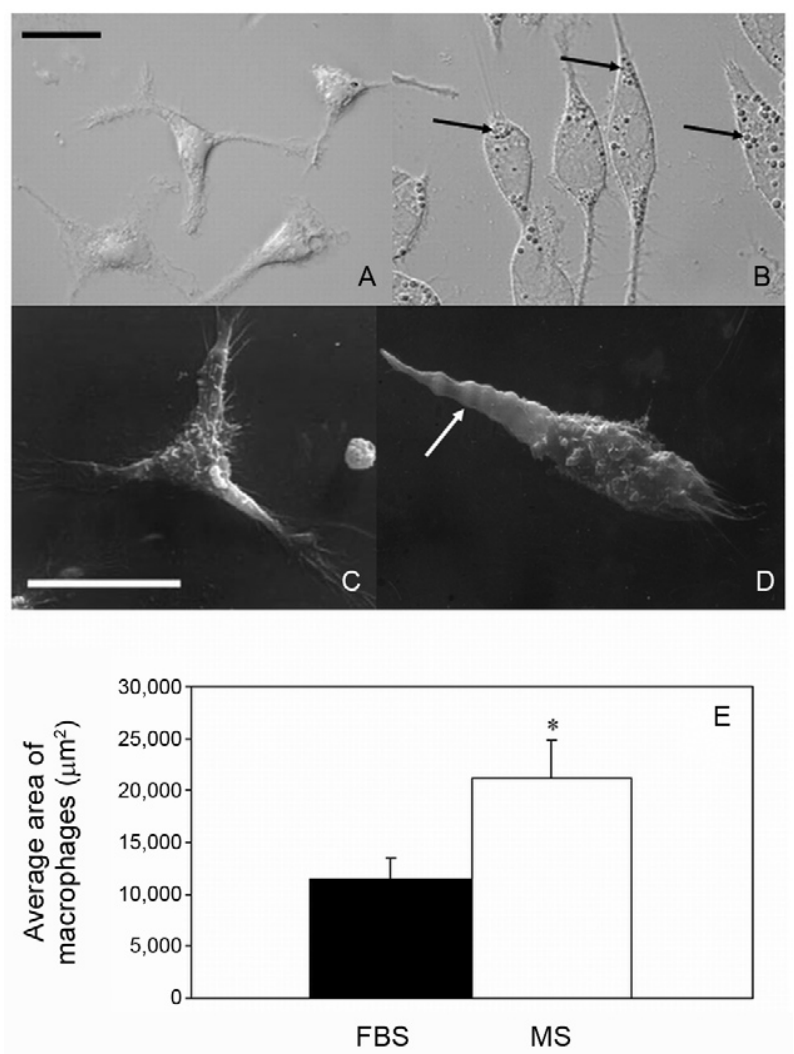

Fig. 1: macrophages cultured with mouse serum (MS) have a different morphology appearance, higher number of vesicle-like organelles and greater area. Macrophages cultured with foetal bovine serum (FBS) (A, C) or MS (B, D). Differential interference contrast microscopy (A, B), Bar $=40 \mu \mathrm{m}$. Scanning electron microscopy $(C, D), B a r=20 \mu \mathrm{m}$. Many vesicles (black arrow) and vesicle contours (white arrow) in macrophages cultured with MS can be seen. Area of macrophages cultured with FBS (black bar) or MS (white bar) of three independent experiments (E). Asterisk means significantly different from values for macrophages cultured with FBS as calculated by the Student $t$ test $(\mathrm{p}>0.05)$.

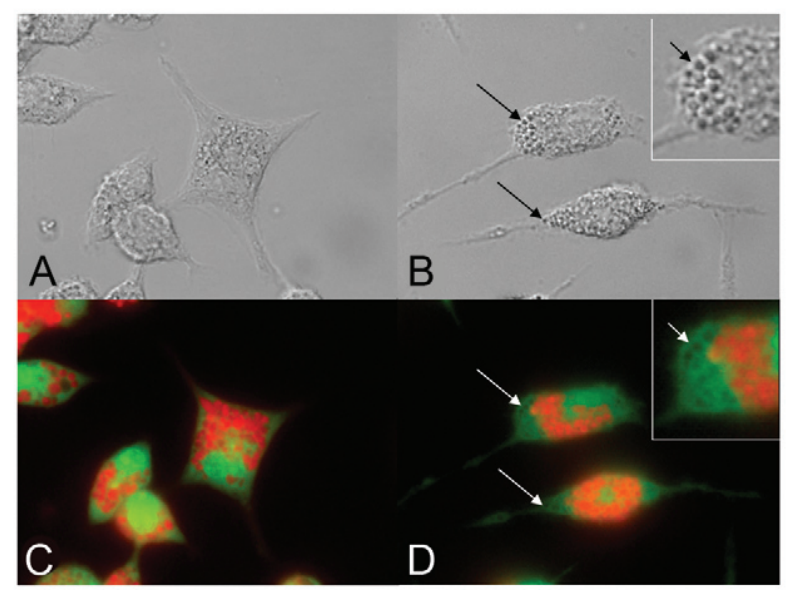

Fig. 2: vesicle-like organelles of macrophages cultured with mouse serum (MS) are not acidic compartments. Differential interference contrast microscopy $(\mathrm{A}, \mathrm{B})$ and the corresponding fluorescent field after acridine orange staining $(C, D)$ of macrophages cultured for $24 \mathrm{~h}$ with foetal bovine serum (A, C) or MS (B, D). Small vesicle-like organelle acridine orange positive can be observed $(C, D)$. However, most of the visible vesicles seen by differential interference contrast microscopy (black arrows) in macrophages cultured with MS (B, D) are acridine orange negative (white arrows). Higher magnification of B and D in the insets. $\mathrm{Bar}=40 \mu \mathrm{m}$. One representative experiment out of three. 

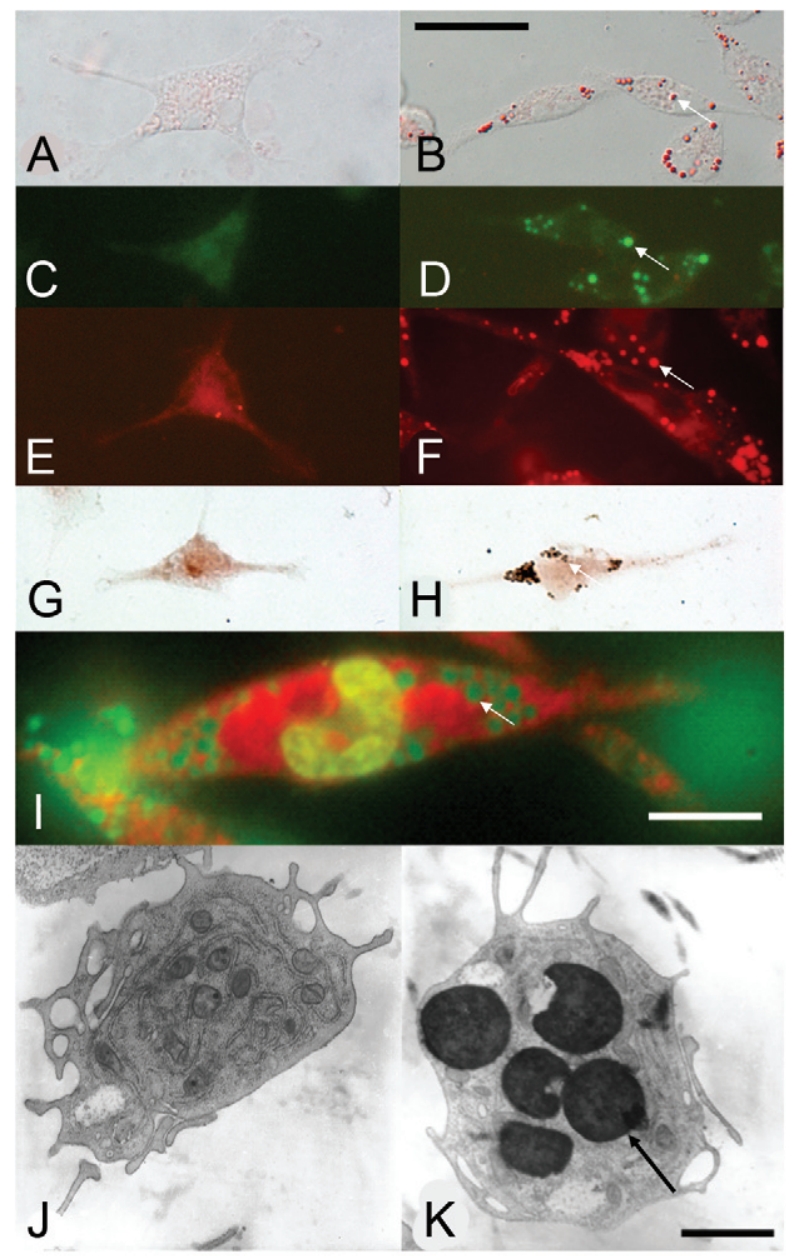

Fig. 3: vesicle-like organelles of macrophages cultured with mouse serum (MS) have a lipidic nature. Bright field microscopy (A, B, G, $\mathrm{H})$, fluorescence microscopy (C-F, I) and transmission electron microscopy after the imidazole-buffered osmium tetroxide method (J, $\mathrm{K}$ ) of macrophages cultured for $24 \mathrm{~h}$ with foetal bovine serum (A, C, E, G, J) or MS (B, D, F, H, I). Oil red (A, B), BODIPY (C, D), Nile red $(\mathrm{E}, \mathrm{F}), \mathrm{OsO}_{4}(\mathrm{G}, \mathrm{H})$ and acridine orange and BODIPY (I) labelling. Culture of macrophages with MS increases the presence of lipid bodies (arrows). Bar $=30 \mu \mathrm{m}$ for A-H, $10 \mu \mathrm{m}$ for I and $1 \mu \mathrm{m}$ for J, K.

confirmed at the ultrastructural level using the imidazolebuffered osmium tetroxide method (Fig. 3J, K).

About half of the fresh peritoneal macrophage population contained LBs as determined by counting LBs after Nile red staining after $1 \mathrm{~h}$ of culture in Hank's solution without sera (Fig. 4) with $1.1 \pm 0.10$ LBs per cell. After $24 \mathrm{~h}$ of culture with $2 \% \mathrm{MS}$, most of the macrophage population had LBs (Fig. 4) and the average number of this organelle per macrophage was $12.0 \pm 2.26$. After 48 $\mathrm{h}$ of culture with MS, the percentage of positive macrophages reached almost $100 \%$ (Fig. 4) and the average number of LBs per macrophage was $16.5 \pm 5.88$. In contrast, culture with $2 \%$ FBS for $24 \mathrm{~h}$ reduced the presence of LBs in the macrophage population (Fig. 4) with an average number of LBs per macrophage of $0.4 \pm 0.24$ and no change in the percentage of macrophages with LBs was detected after $48 \mathrm{~h}$ of culture (Fig. 4) with an average number of this organelle of $0.3 \pm 0.22$. Because

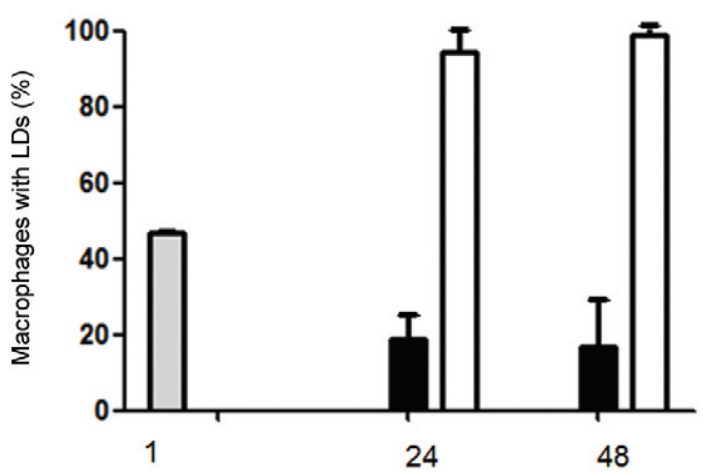

Culture time (h)

Fig. 4: cultured with mouse serum (MS) increases the percentage of macrophages with lipid bodies (LBs). Percentage of macrophages with LBs after culture without (gray bar) and with MS (with bar) or foetal bovine serum (black bar).

lipopolysaccharide induces LB formation (Pacheco et al. 2002), macrophages from the Tlr4-deficient $\mathrm{C} 3 \mathrm{H} /$ HeJ mice were cultured with MS. MS culture induced similar amounts of $\mathrm{LBs}$ in macrophages from $\mathrm{C} 3 \mathrm{H} / \mathrm{HeJ}$ and Swiss mice (data not shown). This result indicates that MS-induced LB formation was independent of lipopolysaccharide contamination.

$L B s$ are associated with the PV of T. gondii - LBs were shown to be associated with tachyzoites in MoMS at $2 \mathrm{~h}$ post-infection (Fig. 5A-C). Tachyzoites were observed in cytoplasmic areas where LBs were located (Fig. 5C). Nile red staining was observed inside the PV containing rosette $24 \mathrm{~h}$ post-infection (Fig. 5D, F). This association was confirmed at the ultrastructural level where LBs were observed in close proximity to the PV and even closely associated to the intracellular parasite in MoMS 6 h post-infection (Fig. 6).

Macrophages cultured in MS showed decreased microbicidal against $T$. gondii - Next, the effect of culturing macrophages in different sera on the production of $\mathrm{NO}$ and $\mathrm{PGE}_{2}$ and their microbicidal capacity against $T$. gondii was determined. Because LBs were present in low numbers in approximately $50 \%$ of the recently obtained macrophages, these cells were cultured for 1, 24 and 48 $\mathrm{h}$ with both sera, classically activated and after $24 \mathrm{~h}$, and $\mathrm{NO}$ and $\mathrm{PGE}_{2}$ production in the culture supernatants were measured. At all three time points, MoMS produced significantly less NO than MoFBS (Fig. 7A). Furthermore, NO production decreased over time independently of the serum used (Fig. 7A). In contrast, $\mathrm{PGE}_{2}$ production in MoMS increased significantly, reaching a production peak when macrophages were cultured for $24 \mathrm{~h}$ (Fig. 7B).

To assess the microbicidal capacity of MoMS and MoFBS and the role of $\mathrm{PGE}_{2}$ production, macrophages were cultured for $24 \mathrm{~h}$, infected with $T$. gondii, activated, treated with indomethacin and further cultured for five days. Then, the microbicidal capacity of these cells was determined by enumerating mechanically released tachyzoites. MoMS were less microbicidal compared to 
MoFBS independent of the activation state (Table). As expected, activation resulted in less tachyzoite growth in both macrophages (Table). Addition of indomethacin did not alter the microbicidal capacity of inactivated macrophages cultured with both sera (Table). However, tachyzoite growth was lower in activated macrophages that were treated with indomethacin (Table).

\section{DISCUSSION}

LBs are lipid-rich, highly heterogeneous, dynamic and regulated organelles. Leukocytes at particular tissue sites receive different stimuli that result in the activation of heterogeneous intracellular signalling pathways that may generate LBs with specific compositions and, thus, distinct functions (Bozza et al. 2011). Here, we described that culture of peritoneal mice macrophages in MS induced morphological changes and the LB formation. Association of LBs with the PV was observed and MoMS produced more $\mathrm{PGE}_{2}$ and less NO. Furthermore, MoMS were less microbicidal to T. gondii and the microbicidal capacity of both macrophages was equivalent upon $\mathrm{PGE}_{2}$ inhibition. These results indicate that culture in MS alters macrophage microbicidal capacity due to higher $\mathrm{PGE}_{2}$ production and low $\mathrm{NO}$ production.

The first observation made after culture of macrophages with MS was the large number of vesicle-like organelles that appeared in these cells. It was clearly demonstrated that the nature of these vesicles were not acidic, but lipidic. This composition was determined us-

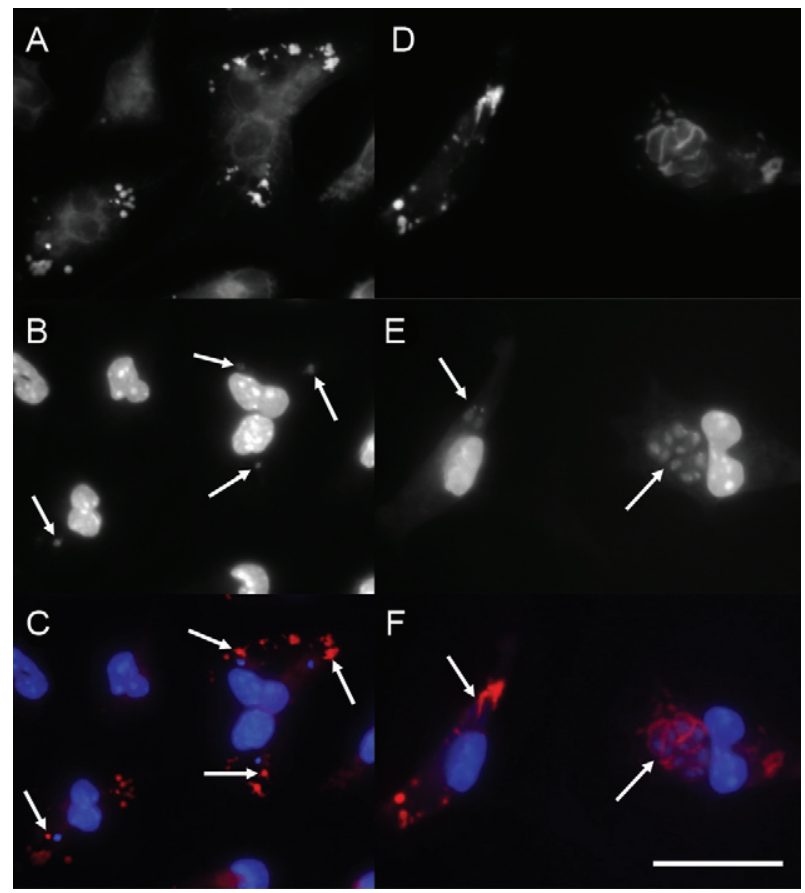

Fig. 5: Toxoplasma gondii associated with lipid bodies (LBs) of infected macrophages cultured with mouse serum. Fluorescent microscopy after Nile red (A, D) and DAPI (B, E) staining after $2 \mathrm{~h}(\mathrm{~A}-\mathrm{C})$ and $24 \mathrm{~h}(\mathrm{D}-\mathrm{F})$ of infection. Merge images (C, F). Note how T. gondii (arrows in B) are located in cytoplasmic areas where LBs (arrows in C) are present right after infection (C). Nile red staining was observed inside the parasitophorous vacuoles after $24 \mathrm{~h}$ of infection $(\mathrm{F})$. Bar $=$ $40 \mu \mathrm{m}$. One representative experiment out of three.

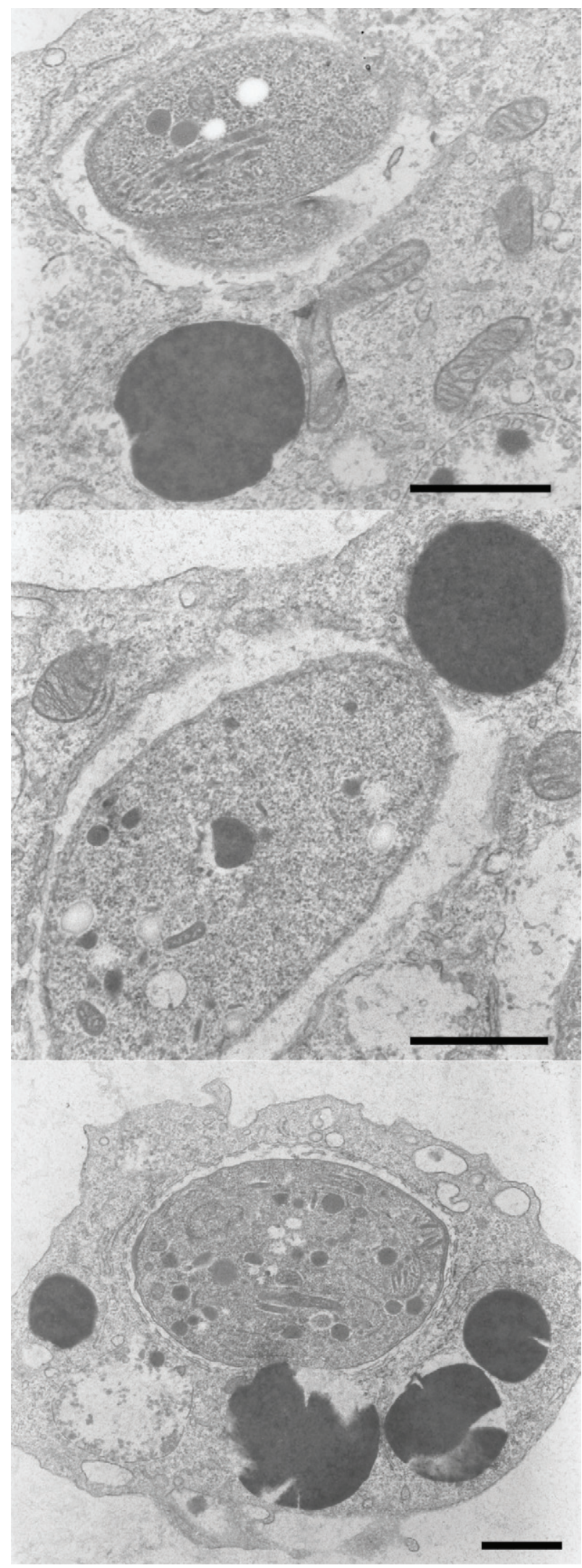

Fig. 6: lipid bodies (LBs) associate to the parasitophorous vacuoles of macrophages infected with Toxoplasma gondii. LBs were evidenced by the imidazole-buffered osmium tetroxide method after culture of macrophages with mouse serum. Note how close LB is to the parasitophorous vacuole. Macrophages were infected for $6 \mathrm{~h}$. Bar $=1 \mu \mathrm{m}$. One representative experiment out of two. 
ing Nile red, oil red, BODIPY and $\mathrm{OsO}_{4}$ staining and double labelling with acridine orange and BODIPY. U1trastructural analyses of macrophages also showed characteristic electron-dense organelles by the imidazolebuffered osmium tetroxide method, clearly indicating that these vesicles were LBs. Furthermore, macrophages exhibited morphological differences when cultured with different sera. Many treatments can change the morphology of macrophages (Jenney \& Anderson 2000). Thus, there must be a specific component of MS that may induce this morphological change. Further experiments
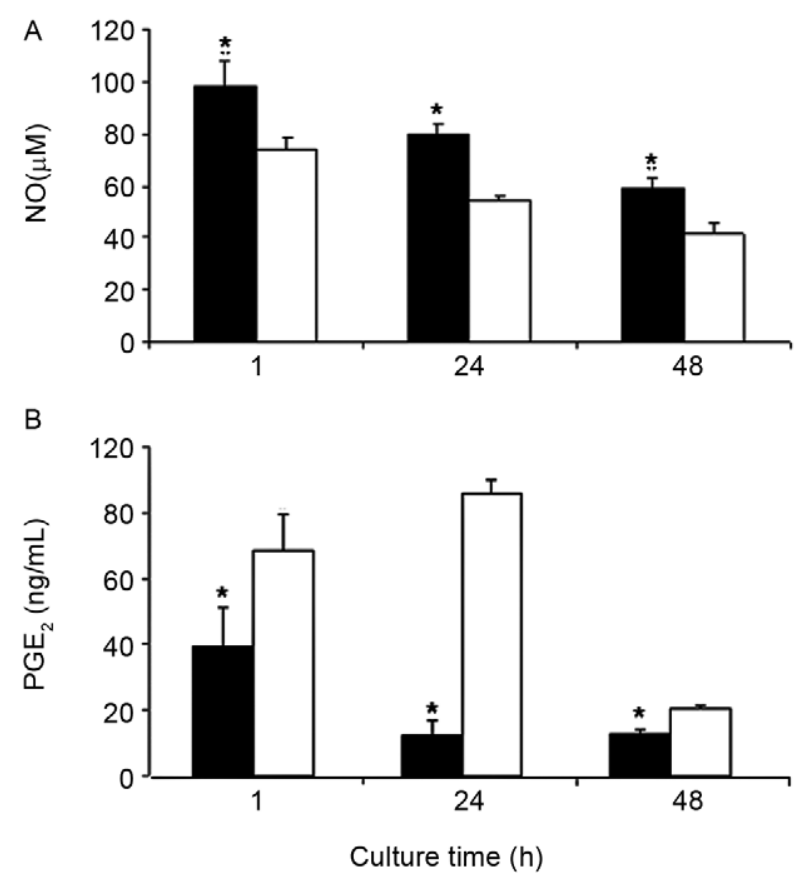

Fig. 7: nitric oxide $(\mathrm{NO})(\mu \mathrm{M})(\mathrm{A})$ and prostaglandin $\mathrm{E}_{2}\left(\mathrm{PGE}_{2}\right)(\mathrm{ng} /$ $\mathrm{mL}$ ) (B) production of activated macrophages cultured with foetal bovine serum (black bars) or mouse serum (MS) (white bars). After 1, 24 or $48 \mathrm{~h}$ of culture, macrophages were activated with interferon- $\gamma$ and lipopolysaccharide and mediators evaluated in the supernatant after $24 \mathrm{~h}$ of culture. Asterisks mean significantly different from respective values for macrophages cultured with MS as calculated by the Student $t$ test $(\mathrm{p}>0.05)$. One representative experiment out of three. concerning the identification of the agents from MS that induce LB formation and cause morphology differences in macrophages are being performed.

Interestingly, pronounced association of LBs with tachyzoites was also observed by light microscopy and ultrastructure analysis. The close proximity of LBs to the PV suggests that $T$. gondii growth may be facilitated by lipids accumulated from the host cell, which would help to explain the higher replication of T. gondii in MoMS. This hypothesis has also been recently suggested for $T$. gondii-infected skeletal muscle cells where LB biogenesis increased and this organelle comes in contact to the PV membrane and the parasite (Gomes et al. 2014). Thus, the association of LBs with the PV may be a recurrent phenomenon in different host cell types. The non-fusogenic PV is known to quickly become physically associated with sites of host cell lipid biosynthesis, the endoplasmic reticulum and mitochondrial membranes (Sinai \& Joiner 1997, 2001). Here, the observed association of the PV with LBs did not exclude its association with either mitochondria or endoplasmic reticulum. Uptake of host lipids by intracellular T. gondii has been reported (de Melo \& de Souza 1996, Charron \& Sibley 2002, Quittnat et al. 2004). In inflamed tissues, leukocytes containing many LBs are observed (Pacheco et al. 2002). Thus, the direct association of LBs with the PV suggests that $T$. gondii may have an advantage because the presence of LBs is an extra energy source and may favour the intracellular survival and growth of this parasite.

The in vitro microbicidal capacity of LB-containing cells has not been tested systematically. Intracellular infection clearly induces the formation of LBs in leukocytes (Bozza et al. 2007). In the cells described here, high numbers of LBs correlated with higher $\mathrm{PGE}_{2}$ production as demonstrated in other cellular models (Pacheco et al. 2002, D’Ávila et al. 2006, Bozza et al. 2007). Thus, the ability of LBs to increase the production of $\mathrm{PGE}_{2}$ that inhibits the T-helper 1 type response (Renz et al. 1988, Betz \& Fox 1991, Freire-de-Lima et al. 2000), including macrophage microbicidal capacity, is well established. Although LBs were not investigated in this system, cultivation of bone marrow mouse macrophages with MS has been demonstrated to increase

TABLE

Microbicidal action against Toxoplasma gondii of activated and non-activated macrophages cultured with foetal bovine serum (FBS) or mouse serum (MS) with or without indomethacin ${ }^{a}$

\begin{tabular}{lccc}
\hline & Indomethacin & FBS & MS \\
\hline Non-activated macrophage & & $71.7 \pm 4.56^{b, c}$ & $124.7 \pm 10.49$ \\
Activated macrophage & + & $19.7 \pm 5.65^{c}$ & $44.3 \pm 14.86^{d}$ \\
Non-activated macrophage & + & $52.3 \pm 15.36^{c}$ & $116.3 \pm 19.56$ \\
Activated macrophage & + & $14.3 \pm 5.03$ & $17.3 \pm 6.81$ \\
\hline
\end{tabular}

$a$ : macrophages were infected with $T$. gondii, activated or not, and cultured for five days with or without indomethacin. Tachyzoites were counted in a Neubauer chamber; $b$ : values are expressed as means and standard deviation in sextuplicate of a representative experiment out of three. Values represent the number of tachyzoites per well after multiplication by $10^{5} ; c$ : significantly different from the respective value for macrophages cultured with MS as calculated by the Student $t$ test ( $\mathrm{p}>0.05)$; $d$ : significantly different from activated macrophages cultured with MS treated with indomethacin as calculated by the Student $t$ test ( $\mathrm{p}>0.05$ ). 
$\mathrm{PGE}_{2}$ production (Shibata 1989). Here we show that the lower microbicidal capacity of MoMS may be related to $\mathrm{PGE}_{2}$ production that most likely down-regulated the NO production. When this eicosanoid was inhibited with indomethacin, MoMS became more microbicidal to $T$. gondii. Thus, a possible mechanism of the lower microbicidal action of MoMS may be that MS induces an intracellular signalling pathway that causes LB formation. The increased number of LBs was followed by an increase in the production of $\mathrm{PGE}_{2}$, which reduces NO production and in turn makes the macrophage less microbicidal. In addition, the high LB content of MoMS may increase the availability of lipids to $T$. gondii, which explains the increased parasite growth. The LB formation induced by pathogen infection has been proposed to be part of the parasite's evasion mechanism (Bozza et al. 2007, Melo \& Dvorak 2012). This hypothesis is sound and should be further investigated.

The use of MS to cultivate cells has proven to be an interesting model to better understand macrophage biology and its heterogeneity. MS has an unknown factor able to induce the expression of the sialoadhesin surface receptor in peritoneal mouse macrophages (Crocker et al. 1988). Other factors that neutralise leukaemia inhibitory factors (Layton et al. 1992) or inhibit growth of some cell lines (CTLL and CTL) (Lindqvist et al. 1992) have also been described in MS. Thus, many factors in MS may be involved in the final macrophage phenotype after culture with this serum, including the appearance of numerous LBs as described here. Further studies are being conducted to characterise the factor in MS that induces LB formation in macrophages. Moreover, culturing macrophages with MS may be a good in vitro model to better understand the function of LBs or other cells that have LBs, such as foam cells.

Culture of mice peritoneal macrophages with MS resulted in a large number of LBs. The MoMS produced less $\mathrm{NO}$ and more $\mathrm{PGE}_{2}$ when compared to MoFBS. In MoMS, LBs associated to were found in close proximity to the PV. These results suggest that MS triggers LB formation in mouse peritoneal macrophages, leading to $\mathrm{PGE}_{2}$ production. This signalling cascade decreases NO production and makes these cells less microbicidal against $T$. gondii, favouring its growth.

\section{ACKNOWLEDGEMENTS}

To Andréa Carvalho César, for proof reading the paper, and to Dr Helene Santos Barbosa, for reagents.

\section{REFERENCES}

Angermüller S, Fahimi HD 1982. Imidazole-buffered osmium tetroxide: an excellent stain for visualization of lipids in transmission electron microscopy. Histochem J 14: 823-835.

Beller M, Thiel K, Thul PJ, Jäckle H 2010. Lipid droplets: a dynamic organelle into focus. FEBS Lett 584: 2176-2182.

Betz M, Fox BS 1991. Prostaglandin E inhibits production of Th1 lymphokines, but not of Th2 lymphokines. J Immunol 146: 108-113.

Bozza PT, Bakker-Abreu I, Navarro-Xavier RA, Bandeira-Melo C 2011. Lipid body function in eicosanoid synthesis: an update. Prostaglandins Leukot Essent Fatty Acids 85: 205-213.

Bozza PT, Magalhães KG, Weller PF 2009. Leukocyte lipid bodies - biogenesis and functions in inflammation. Biochim Biophys Acta 1791: 540-551.
Bozza PT, Melo RC, Bandeira-Melo C 2007. Leukocyte lipid bodies regulation and function: contribution to allergy and host defense. Pharmacol Ther 113: 30-49.

Caffaro CE, Boothroyd JC 2011. Evidence for host cells as the major contributor of lipids in the intravacuolar network of Toxoplasmainfected cells. Eukaryot Cell 10: 1095-1099.

Charron AJ, Sibley LD 2002. Host cells: mobilizable lipid resources for the intracellular parasite Toxoplasma gondii. J Cell Sci 115: 3049-3059.

Coppens I 2006. Contribution of host lipids to Toxoplasma pathogenesis. Cell Microbiol 8: 1-9.

Crocker PR, Hill M, Gordon S 1988. Regulation of a murine macrophage haemagglutinin (sheep erythrocyte receptor) by a species-restricted serum factor. Immunology 65: 515-522.

D’Ávila H, Freire-de-Lima CG, Roque NR, Teixeira L, Barja-Fidalgo C, Silva AR, Melo RC, dos Reis GA, Castro-Faria-Neto HC, Bozza PT 2011. Host cell lipid bodies triggered by Trypanosoma cruzi infection and enhanced by the uptake of apoptotic cells are associated with prostaglandin $\mathrm{E}_{2}$ generation and increased parasite growth. J Infect Dis 204: 951-961.

D’Ávila H, Melo RC, Parreira GG, Werneck-Barroso E, CastroFaria-Neto HC, Bozza PT 2006. Mycobacterium bovis bacillus Calmette-Guérin induces TLR2-mediated formation of lipid bodies: intracellular domains for eicosanoid synthesis in vivo. J Immunol 176: 3087-3097.

Dahl CA, Lindqvist C 1989. Effects of normal mouse serum on the IL3-induced proliferation of bone marrow cells. Blood 73: 700-705.

de Melo EJ, de Souza W 1996. Pathway of C6-NBD-ceramide on the host cell infected with Toxoplasma gondii. Cell Struct Funct 21: 47-52.

Freire-de-Lima CG, Nascimento DO, Soares MB, Bozza PT, CastroFaria-Neto HC, de Mello FG, dos Reis GA, Lopes MF 2000. Uptake of apoptotic cells drives the growth of a pathogenic trypanosome in macrophages. Nature 403: 199-203.

Gomes AF, Magalhães KG, Rodrigues RM, de Carvalho L, Molinaro R, Bozza PT, Barbosa HS 2014. Toxoplasma gondii-skeletal muscle cells interaction increases lipid droplet biogenesis and positively modulates the production of IL-12, IFN-g and $\mathrm{PGE}_{2}$. Parasit Vectors 7: 47.

Gordon S, Taylor PR 2005. Monocyte and macrophage heterogeneity. Nat Rev Immunol 5: 953-964.

Guillermo LV, DaMatta RA 2004. Nitric oxide inhibition after Toxoplasma gondii infection of chicken macrophage cell line. Poult Sci 83: 776-782.

Jenney CR, Anderson JM 2000. Adsorbed serum proteins responsible for surface dependent human macrophage behavior. $J$ Biomed Mater Res 49: 435-447.

Layton MJ, Cross BA, Metcalf D, Ward LD, Simpson RJ, Nicola NA 1992. A major binding protein for leukemia inhibitory factor in normal mouse serum: identification as a soluble form of the cellular receptor. Proc Natl Acad Sci USA 89: 8616-8620.

Lindqvist C, Dahl C, Back C, Oker-Blom C, Akerman K, Wigzell H 1992. Normal mouse serum-derived factor(s) which inhibits growth of the interleukin-2-dependent cell line CTLL. Eur $J$ Haematol 49: 36-45.

Martinez FO, Helming L, Gordon S 2009. Alternative activation of macrophages: an immunological function perspective. Annu Rev Immunol 27: 451-483.

Melo RC, D’Ávila H, Bozza PT, Weller PF 2011. Imaging lipid bodies within different light microscopy techniques. Methods Mol Biol 689: 149-161. 
Melo RC, Dvorak AM 2012. Lipid body-phagosome interaction in macrophages during infectious diseases: host defense or pathogen survival strategy? PLoS Pathog 8: e1002729.

Melo RCN, Fabrino DL, Dias FF, Parreira GG 2006. Lipid bodies: structural markers of inflammatory macrophages in innate immunity. Inflamm Res 55: 342-348.

Monteiro VG, Lobato CSS, Silva AR, Medina DV, Oliveira MA, Seabra SH, de Souza W, DaMatta RA 2005. Increased association of Trypanosoma cruzi with silaloadhesin positive mice macrophages. Parasitol Res 97: 380-385.

Nishikawa Y, Quittnat F, Stedman TT, Voelker DR, Choi JY, Zahn M, Yang M, Pypaert M, Joiner KA, Coppens I 2005. Host cell lipids control cholesteryl ester synthesis and storage in intracellular Toxoplasma. Cell Microbiol 7: 849-867.

Pacheco P, Bozza FA, Gomes RN, Bozza M, Weller PF, Castro-FariaNeto HC, Bozza PT 2002. Lipopolysaccharide-induced leukocyte lipid body formation in vivo: innate immunity elicited intracellular loci involved in eicosanoid metabolism. J Immunol 169: 6498-6506.

Quittnat F, Nishikawa Y, Stedman TT, Voelker DR, Choi JY, Zahn MM, Murphy RC, Barkley RM, Pypaert M, Joiner KA, Coppens I 2004. On the biogenesis of lipid bodies in ancient eukaryotes: synthesis of triacylglycerols by a Toxoplasma DGAT1-related enzyme. Mol Biochem Parasitol 138: 107-122.
Renz H, Gong JH, Schmidt A, Nain M, Gemsa D 1988. Release of tumor necrosis factor-alpha from macrophages. Enhancement and suppression are dose-dependently regulated by prostaglandin $\mathrm{E}_{2}$ and cyclic nucleotides. J Immunol 141: 2388-2393.

Salucci V, Lena AM, Ciliberto G, Scarselli E, La Monica N 2005. Adenovirus transduction and culture conditions affect the immunogenicity of murine dendritic cells. Scand J Immunol 62: 206-217.

Samsa MM, Mondotte JA, Iglesias NG, Assunção-Miranda I, Barbosa-Lima G, da Poian AT, Bozza PT, Gamarnik AV 2009. Dengue virus capsid protein usurps lipid droplets for viral particle formation. PLoS Pathog 5: e1000632.

Seabra SH, de Souza W, DaMatta RA 2004. Toxoplasma gondii exposes phosphatidylserine inducing a TGF-betal autocrine effect orchestrating macrophage evasion. Biochem Biophys Res Commun 324: 744-752.

Shibata Y 1989. Humoral factors in the induction of prostaglandin $E_{2}-$ producing macrophages in vitro. Reg Immunol 2: 158-168.

Sinai AP, Joiner KA 1997. Safe haven: the cell biology of non-fusogenic pathogen vacuoles. Annu Rev Microbiol 51: 415-462.

Sinai AP, Joiner KA 2001. The Toxoplasma gondii protein ROP2 mediates host organelle association with the parasitophorous vacuole membrane. J Cell Biol 154: 95-108. 